\title{
HALLAZGO DE HEPATOZOON CANIS EN CANINOS (CANIS FAMILIARIS) EN LA CIUDAD DE ESPERANZA, SANTA FE (ARGENTINA)
}

\author{
Ruiz, M. F. ${ }^{1} ;$ Zimmermann, R. N. ${ }^{1}$; AguiRRe, F. O. ${ }^{1}$; \\ BONO, M. F. ${ }^{2} \&$ WIDENHORN, N. I. ${ }^{3}$
}

\begin{abstract}
RESUMEN
La hepatozoonosis es una enfermedad infecciosa de origen parasitario producida por Hepatozoon canis (James, 1905). El primer reporte de este hemoparásito se realizó en la India en 1905, en Argentina se describe por primera vez en 1999. Se transmite principalmente por la ingestión de la garrapata Rhipicephalus sanguineus. La mayoría de los cánidos afectados tienen una infección subclínica, aquellos que desarrollan signología generalmente son jóvenes, menores de 4 meses, inmunodeficientes o con enfermedades concurrentes.

El objetivo de este trabajo es comunicar la presencia de $H$. canis en caninos que residen en la ciudad de Esperanza, Santa Fe, Argentina. La presencia de este parásito no ha sido reportada con anterioridad en la zona de influencia de la Facultad de Ciencias Veterinarias, UNL.

Palabras claves: Hepatozoon canis, caninos, Esperanza, Santa Fe, Argentina.
\end{abstract}

\section{SUMMARY}

Find of Hepatozoon canis in dogs (Canis familiaris) of Esperanza, Santa Fe, Argentina.

The hepatozoonosis is an infectious disease of parasitic origin, produced by Hepatozoon canis (James, 1905). The first report of this hemoparasite was performed in India in 1905, in Argentina it was described for the first time in 1999. It is mostly transmitted by the ingestion of the tick Rhipicephalus sanguineus. Most of the affected dogs have a subclinical infection those that develop symptoms are usually young under four months, inmunodeficient or with coincident disease. The

1.- Laboratorio de Análisis Clínicos. Hospital de Salud Animal. Facultad de Ciencias Veterinarias (UNL). Kreder 2805. (3080) Esperanza, provincia de Santa Fe.

2.- Laboratorio de Estudios Parasitológicos. Cátedra de Parasitología y Enfermedades Parasitarias. FCV

(UNL).

3.- Prácticas Hospitalarias de Pequeños Animales. Hospital de Salud Animal. FCV (UNL).

Manuscrito recibido el 23 de octubre de 2012 y aceptado para su publicación el 4 de febrero de 2013. 
objective of this work is to communicate the presence of $H$. canis in dogs that live in the town of Esperanza, Santa Fe, Argentina. Presence of this parasite has not been previously reported in the area under the influence of Facultad de Ciencias Veterinarias, Universidad Nacional del Litoral.

Key words: Hepatozoon canis, dogs, Esperanza, Santa Fe, Argentina

\section{INTRODUCCIÓN}

La hepatozoonosis es una enfermedad infecciosa de origen parasitario. El agente etiológico es un protozoo apicomplexo perteneciente a la clase Sporozoea, orden Eucoccidia, Familia Haemogregarinidae, género Hepatozoon (Cordero del Campillo \& Rojo Vázquez, 2000; Mateus Ardila et al., 2007).

La bibliografía cita más de trescientas especies diferentes de Hepatozoon que parasitan a anfibios, reptiles, aves y mamíferos. Los que parasitan a anfibios, reptiles y aves se localiza en los eritrocitos, mientras que en los mamíferos se encuentra parasitando a los leucocitos, principalmente neutrófilos y monocitos (Greene, 2008). En los caninos se han identificado dos especies (Mora et al., 2004; Greene, 2008): 1) H. americanum, típico de Estados Unidos y que es transmitido por la garrapata Amblyomma maculatum (Ewing \& Panciera, 2003). 2) H. canis, aislada en África, Asia, sur de Europa y algunos países sudamericanos, como Colombia (Mateus Ardila et al., 2007), Venezuela, Brasil y Argentina (Mora et al., 2004; Greene, 2008).

El H. canis fue reportado por primera vez en la India en el año 1905 (Kujman et al., 2005; Mateus Ardila et al., 2007). En Argentina se describió por primera vez en el año 1999 (Silva et al., 1999) y desde entonces los hallazgos se han incrementados, habiéndose reportado en las provincias de Buenos Aires (Pérez Tort et al., 2007; Eiras et al., 2010), Chubut (Trelew), Mendoza,
Salta (El Carril), San Luis, Santa Fe (Rosario) (Linares, 2011).

El $H$. canis se transmite principalmente por la ingestión de la garrapata común del perro, Rhipicephalus sanguineus infectada con el parásito. En cachorros se describe la transmisión vertical (Cordero del Campillo \& Rojo Vázquez, 2000; Kujman et al., 2005; Greene, 2008).

El ciclo biológico de este parásito se divide en dos etapas: la primera se produce dentro de la garrapata y la segunda en los caninos. La garrapata se infecta durante la ingestión de sangre sobre un hospedador infectado al ingerir gametocitos presentes en los neutrófilos o monocitos. El parásito se multiplica hasta esporozoíto en el hematocele sin migrar a las glándulas salivales. (Soulsby, 1987).

Después de que el perro ingiere una garrapata infectada, los esporozoítos son liberados en la luz intestinal, penetran la pared del mismo y son llevados vía sanguínea o linfática para localizarse en células endoteliales y células fagocíticas. Dentro de éstas células tiene lugar la fase de merogonia con formación de merontes, que dependiendo de su posterior evolución se clasifican en macromerontes y micromerontes. Los micromerontes dan lugar a la formación de pequeños merozoítos que al liberarse y ser fagocitados forman los gametocitos, estadio que se visualiza en sangre periférica y del que nos valemos para el diagnóstico (Jáurregui Latorre \& Lopéz Girón, 1995). El ciclo se reinicia cuando una garrapata ingiere los leucocitos infectados. 